\title{
Formation and destruction of jets in X-ray binaries
}

\author{
N. D. Kylafis ${ }^{1,2}$, I. Contopoulos ${ }^{3}$, D. Kazanas ${ }^{4}$, and D. M. Christodoulou ${ }^{5}$ \\ 1 University of Crete, Physics Department \& Institute of Theoretical \& Computational Physics, 71003 Heraklion, Crete, Greece \\ e-mail: kylafis@physics.uoc.gr \\ 2 Foundation for Research and Technology-Hellas, 71110 Heraklion, Crete, Greece \\ 3 Research Center for Astronomy, Academy of Athens, 11527 Athens, Greece \\ ${ }^{4}$ NASA/GSFC, Code 663, Greenbelt, MD 20771, USA \\ 5 Department of Mathematical Sciences, University of Massachusetts Lowell, Lowell, MA 01854, USA
}

Received 8 April 2011 / Accepted 15 November 2011

\begin{abstract}
Context. Neutron-star and black-hole X-ray binaries (XRBs) exhibit radio jets, whose properties depend on the X-ray spectral state and history of the source. In particular, black-hole XRBs emit compact, steady radio jets when they are in the so-called hard state. These jets become eruptive as the sources move toward the soft state, disappear in the soft state, and then re-appear when the sources return to the hard state. The jets from neutron-star X-ray binaries are typically weaker radio emitters than the black-hole ones at the same X-ray luminosity and in some cases radio emission is detected in the soft state.

Aims. Significant phenomenology has been developed to describe the spectral states of neutron-star and black-hole XRBs, and there is general agreement about the type of the accretion disk around the compact object in the various spectral states. We investigate whether the phenomenology describing the X-ray emission on one hand and the jet appearance and disappearance on the other can be put together in a consistent physical picture.

Methods. We consider the so-called Poynting-Robertson cosmic battery (PRCB), which has been shown to explain in a natural way the formation of magnetic fields in the disks of AGNs and the ejection of jets. We investigate whether the PRCB can also explain the formation, destruction, and variability of jets in XRBs.

Results. We find excellent agreement between the conditions under which the PRCB is efficient (i.e., the type of the accretion disk) and the emission or destruction of the radio jet.

Conclusions. The disk-jet connection in XRBs can be explained in a natural way using the PRCB.
\end{abstract}

Key words. stars: neutron - accretion, accretion disks - black holes physics - X-rays: binaries - magnetic fields

\section{Introduction}

Jets have been observed from both neutron-star and blackhole X-ray binaries (XRBs). There have been many studies of the theory of jets and outflows off disks of compact objects (e.g., Blandford \& Payne 1982; Meier 2001, 2005; Blandford \& Begelman 2004; Machida et al. 2006; Fereira et al. 2006; Fragile \& Meier 2009; Spruit 2010, and many others). A vast amount of phenomenology has also been developed regarding accretion and jets in black-hole XRBs as summarized in Sect. 3 below (for reviews see Fender et al. 2004a; Homan \& Belloni 2005; Remillard \& McClintock 2006; Done et al. 2007; Fender et al. 2009; Belloni 2010; Markoff 2010). Neutron-star jets are typically fainter radio emitters than their black-hole counterparts at the same X-ray luminosity (Fender \& Kuulkers 2001; Migliari \& Fender 2006; Migliari et al. 2007; Tudose et al. 2009; Migliari et al. 2010), thus they have been studied less.

A key point missing in the aforementioned rich phenomenology is a physical explanation of the jet appearance, disappearance, and re-appearance as the sources follow a "q"shaped curve in the so-called hardness-intensity diagram (HID, first introduced in the present context by Miyamoto et al. 1995; Fig. $1^{1}$ ). We demonstrate that by invoking a simple

\footnotetext{
1 The figure is publicly available from http://www . issibern. ch/teams/proaccretion/Documents. html
}

physical mechanism proposed more than ten years ago, the so-called Poynting-Robertson cosmic battery (hereafter PRCB; Contopoulos \& Kazanas 1998), we can explain in a natural way many of the manifestations of the jets. Our presentation is semiquantitative and we postpone detailed calculations to the future. Here we simply aim to outline the general scheme. In Sect. 2 we describe briefly how this mechanism works, in Sect. 3 we view jet formation and destruction in the context of the PRCB, and we explain the differences between neutron-star and black-hole jets, and in Sect. 4 we present our conclusions and our theoretical predictions.

\section{The origin of the magnetic field: a cosmic battery}

The PRCB was originally proposed as a means to account for the origin of magnetic fields in the accretion flows surrounding astrophysical compact objects (Contopoulos \& Kazanas 1998; see also Contopoulos et al. 2006; Christodoulou et al. 2008). This physical mechanism has found observational support in observations of the Faraday rotation-measure gradients across extragalactic jets (Contopoulos et al. 2009; Christodoulou et al. 2011). The PRCB is based on the Poynting-Robertson drag effect on the electrons of the innermost plasma orbiting a black hole or neutron star. We describe here how the PRCB works in the case of a stellar-mass black hole in an XRB, and we discuss differences in the case of a neutron star in Sect. 3.2. 


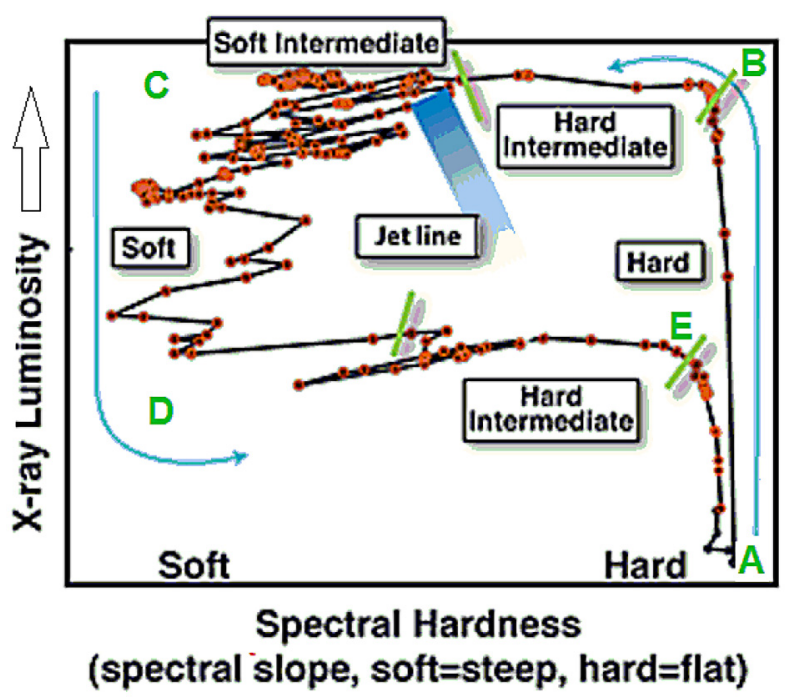

Fig. 1. Schematic representation of the "q"-shaped curve in a hardnessintensity diagram for black-hole X-ray binaries (see, e.g., Belloni 2010).

It is well-known that the accretion disk around a black hole does not extend all the way to the event horizon, but is truncated at an inner radius owing to the existence of an innermost stable circular orbit (hereafter ISCO). As the accretion disk material gradually spirals in towards the ISCO, it emits blackbody radiation at increasingly higher temperatures reaching $\sim 10^{7} \mathrm{~K}$ at the ISCO. Within this radius, the material is essentially in free fall and emits very little radiation.

Electrons in the plasma orbiting the black hole at the inner edge of the accretion disk scatter photons coming from the opposite side of the disk inner edge. As they circle the black hole with a speed comparable to the speed of light, they see the photons aberrated as if they were coming from a slightly head-on direction. Thus, the electrons experience a force against their direction of motion ${ }^{2}$. The ions feel a much weaker radiation-drag force than the electrons because the Thompson cross-section is inversely proportional to the square of the mass of the scatterer. This difference in the radiation-drag force leads to an increase in the relative motion between electrons and ions that is equivalent to an increase in the electric current in the direction of rotation, hence a stronger poloidal magnetic field. When one extends the MHD equations to incorporate the equations of motion of the electron and ion fluids, one sees that the growth of the axial magnetic field is associated with an azimuthal electromotive field $\boldsymbol{E}_{\mathrm{PR}}$ in the direction opposite that of rotation at the inner edge of the accretion flow (Contopoulos \& Kazanas 1998). There is little or no azimuthal electromotive field within the ISCO (where the plasma freely plunges) or at larger radii in the accretion disk (since the optical depth increases and the radiation flux decreases rapidly with distance). The growth of the axial magnetic field around the inner edge of the accretion flow is driven by the nonzero curl of the distribution of azimuthal electromotive field $\boldsymbol{E}_{\mathrm{PR}}$ according to the induction equation

$\frac{\partial B}{\partial t}=-c \nabla \times\left.\boldsymbol{E}_{\mathrm{PR}}\right|_{z}=-\frac{c}{r} \frac{\partial}{\partial r}\left(r E_{\mathrm{PR}}\right)$

2 The aberration of radiation is the essence of the Poynting-Robertson effect felt by grains in orbit around the Sun. Contopoulos \& Kazanas (1998) applied the same principle to the electrons in a plasma orbiting an astrophysical compact object, assuming an isotropic source of radiation at the center. In what follows, we relax the assumption of isotropy. A detailed calculation of the aberration effect in the relativistic environment of a black hole is in progress. where $B$ is the value of the axial $(z)$ magnetic field inside the ISCO, $c$ is the speed of light, and a cylindrical system of coordinates $(r, \phi, z)$ centered on the central compact object and aligned with the direction of rotation of the system is used. We note that the advection and diffusion terms of Eq. (5) in Contopoulos \& Kazanas (1998) were ignored in Eq. (1) to avoid complicating the presentation. One can further simplify Eq. (1) by considering the magnetic vector potential $\boldsymbol{A}$, defined through $\boldsymbol{B}=\nabla \times \boldsymbol{A}$, and in particular its $\phi$ component $A_{\phi} \equiv \Psi /(2 \pi r)$, where $\Psi$ is the total poloidal magnetic flux accumulated inside radius $r$. Equation (1) can then be written as

$\frac{\partial \Psi}{\partial t}=-2 \pi r c E_{\mathrm{PR}}$

Preliminary global axisymmetric ideal MHD numerical simulations performed by Christodoulou et al. (2008) showed that this effect does indeed take place around compact astrophysical objects. The problem with these simulations is that they need to follow both the short dynamical timescale at the inner edge of the disk, and the large-scale evolution timescale for the growth of the magnetic field to dynamically significant values (see below). To observe the PRCB effect within the integration time available in our original preliminary simulations (on the order of a few hundred inner-disk dynamical timescales), $E_{\mathrm{PR}}$ was artificially amplified by several orders of magnitude. Current supercomputer power allows us, in principle, to integrate for several million inner-disk dynamical timescales (Tchekhovskoy, priv. comm.), which will allow us in the future to perform numerical simulations of the PRCB with realistic values of $E_{\mathrm{PR}}$.

Equation (2) does not take into account the back-reaction of the resulting magnetic field on the dynamics of the plasma in orbit around the compact object. The magnetic field can increase in strength to a maximum value dictated by equipartition with the kinetic energy of the plasma at the inner edge of the accretion disk, which, for reasons of simplicity, is assumed to lie at the position of the ISCO of a Schwarzchild (i.e. non-rotating) black hole (the general case of a spinning black hole in which the ISCO can lie all the way down to the horizon being currently under investigation)

$$
r=6 G M / c^{2} \sim 10\left(\frac{M}{M_{\odot}}\right) \mathrm{km} .
$$

We can then estimate the equipartition magnetic field as

$$
\begin{aligned}
B_{\mathrm{eq}} & \sim\left(4 \pi \rho v_{\mathrm{r}} v_{\mathrm{K}}\right)^{1 / 2} \sim\left(\frac{2 \dot{M} v_{\mathrm{K}}}{r h}\right)^{1 / 2} \\
& \sim 10^{8} \dot{m}^{1 / 2}\left(\frac{h}{r}\right)^{-1 / 2}\left(\frac{M}{M_{\odot}}\right)^{-1 / 2} \mathrm{G},
\end{aligned}
$$

where $\rho$ is the density, $v_{\mathrm{r}}$ and $v_{\mathrm{K}}$ are the radial and the Keplerian velocities, respectively, and $\dot{m}$ is the mass accretion rate in units of its Eddington value $\dot{M}_{\mathrm{E}} \equiv L_{\mathrm{E}} / c^{2}$, and all quantities have been evaluated at the ISCO. The above calculation is only one of several possible ways to estimate $B_{\text {eq }}$. Other formulations may consider the total (gas plus ram) pressure or even the tension of the field (see footnote 3). These estimates differ from those obtained with Eq. (4) by factors of order "a few". As described below, our main interest does not lie in the particular value of the equipartition field, but in the order of magnitude of the characteristic time that the PRCB takes to grow the field to near equipartition. 
At early times, Eq. (2) applies continuously, and the growth of the magnetic field is linear with a characteristic timescale $\tau_{\mathrm{eq}}$ to reach equipartition equal to

$\tau_{\mathrm{eq}} \sim \frac{r B_{\mathrm{eq}}}{2 c E_{\mathrm{PR}}}$.

To complete our calculation, we need to estimate the PRCB electromotive field $E_{\mathrm{PR}}$. As we have said, this calculation requires a detailed consideration of photon orbits in the relativistic environment of a black hole. Here we estimate this term geometrically from the radiation that an electron at the ISCO sees coming from the "wall" of height $h$ at the opposite side of the inner edge of the accretion flow. The total luminosity emitted is

$$
\begin{aligned}
L & \sim f \sigma T^{4} \pi r h \\
& \sim 10^{36} f T_{7}^{4}\left(\frac{M}{M_{\odot}}\right)^{2}\left(\frac{h}{r}\right) \mathrm{erg} \mathrm{s}^{-1},
\end{aligned}
$$

where $T_{7}$ is the ISCO equivalent blackbody temperature in units of $10^{7} \mathrm{~K}, \sigma$ is the Stefan-Boltzmann constant, and we include the geometric factor $f$ of order unity because part of the radiation coming from the opposite side of the flow falls into the black hole and does not reach the other side. The PRCB electromotive field can now be estimated as

$$
E_{\mathrm{PR}} \sim g \frac{L \sigma_{\mathrm{T}}}{4 \pi r^{2} c e} \frac{v_{\mathrm{K}}}{c},
$$

where $\sigma_{\mathrm{T}}$ is the electron Thomson cross-section, $v_{\mathrm{K}}$ is the Keplerian velocity at the ISCO, $v_{\mathrm{K}} / c$ is the Poynting-Robertson aberration effect caused by photons "hitting" the moving electrons on the other side at about $90^{\circ}$, and $g$ is a geometric factor of order unity that accurately accounts for light bending in the immediate environment of the central black hole. Substituting these expessions into Eq. (5), we obtain

$\tau_{\text {eq }} \sim 4 \times 10^{5}(f g)^{-1} T_{7}^{-4} \dot{m}^{1 / 2}\left(\frac{h}{r}\right)^{-3 / 2}\left(\frac{M}{M_{\odot}}\right)^{1 / 2} \mathrm{~s}$.

We recall that Contopoulos \& Kazanas (1998) obtained a simpler estimate of the above timescale under the simplifying assumption that the source luminosity originates from the center, namely $\tau_{\mathrm{eq}} \sim 4 \times 10^{-5} \dot{m}^{-1 / 2}\left(M / M_{\odot}\right)^{3 / 2}$ yr. Our present, more elaborate calculation, elucidates the dependence of the PRCB timescale on the physical state of the accretion disk (thick versus thin), which seems to be of paramount importance in the phenomenon of jet formation and destruction in XRBs (see Sect. 3).

To reach a current strong enough to produce the magnetic field on the order of $10^{6} \mathrm{G}(\sim 10 \%$ of the equipartition value) required to explain the radio emission in a $10 M_{\odot}$ source such as Cyg X-1 (Giannios 2005), the battery must operate for about $12 \mathrm{~h}$, if the inner part of the accretion flow is described by an ADAF with $h / r \sim 1$ and $\dot{m} \sim 10^{-1}$. This timescale is short compared to the system evolution timescale up to the first crossing of the jet line (see Fig. 1) or after the second crossing of the jet line at lower X-ray luminosity (as an example, we refer to the observed timescales for the 300-day outburst of J1752-223 in Stiele et al. 2011).

However, when the system moves from the hard to the soft state, the accretion flow changes from a thick ADAF to a Shakura-Sunyaev thin disk with $h / r \sim 10^{-1}$. In this case, the timescale for the growth of a $10^{6} \mathrm{G}$ magnetic field ( $\sim 3 \%$ of the equipartition value) becomes on the order of five days (assuming that $\dot{m}$ is still $\sim 10^{-1}$ ), which is longer than the characteristic evolution timescale of the black-hole XRB in the soft state (see the evolution past region $\mathrm{C}$ in Fig. 1, Sect. 3.1 below, and Fig. 4 of Stiele et al. 2011).

\section{Jet formation and destruction}

A vast amount of work done in three-dimensional (3D) magnetohydrodynamic (MHD) simulations of astrophysical accreting systems (e.g. Machida et al. 2006; Hawley 2009; Romanova et al. 2009; Mignone et al. 2010, and references therein) have apparently shown that jet formation and acceleration is due to one of two types of physical mechanisms: plasma gun/magnetic towers, as proposed by Contopoulos (1995)/Lynden-Bell (1996), or centrifugal driving, as proposed by Blandford \& Payne (1982). What is most important, however, is that these simulations demostrate that at the origin of jet formation and acceleration lies a large-scale magnetic field that threads the central "driving engine" (the central compact object and the surrounding innermost accretion disk). Once this field is in place, jet formation can proceed through any one of the above mechanisms. In contrast, without an established large-scale magnetic field, jet formation is impossible.

Most early simulations of MHD jet formation did not investigate the origin of the required large-scale magnetic field (it was simply assumed as an initial/boundary condition). Were the disk material to be infinitely conducting, accretion would naturally advect and amplify any weak magnetic field present on large scales. Magnetorotational instability (MRI) simulations, however, suggest that astrophysical accretion disks develop turbulent magnetic diffusivities comparable to their turbulent viscosities, indicating that weak large-scale magnetic fields rapidly diffuse outward (e.g. Lubow et al. 1994). Numerical simulations have shown that, under certain physical conditions, advection succeeds in establishing a strong magnetic field around the central object (e.g. Igumenshchev 2008; Lovelace et al. 2009; Tchekhovskoy et al. 2011). We are currently investigating these conditions.

In the present paper, we consider an independent physical scenario and associate the origin of the magnetic field with the PRCB. In what follows, we assume that, whenever the PRCB operates efficiently, a large-scale magnetic field is generated in the immediate vicinity of the central compact object, which, according to previous 3D MHD simulations, leads to the formation of jets. In the opposite limit, when the PRCB operates inefficiently, the resulting magnetic field is weaker, as is the jet power and emission. Therefore, under the assumption that the PRCB is in operation in XRBs, we examine whether a consistent picture can be drawn that explains all the manifestations of the jets in these sources.

\subsection{Jets in black-hole $X$-ray binaries}

In the HID, a steady jet exists even when the sources are in the so-called quiescent state, where the spectrum is dominated by hard X-rays, and typically $L_{\mathrm{X}} \sim 10^{-8}-10^{-6} L_{\mathrm{E}}$ (Kong et al. 2002; Hameury et al. 2003; Remillard \& McClintock 2006; Gallo et al. 2006). To be more accurate, the "q"-shaped curve shown in Fig. 1 may turn to the left at its bottom right part, i.e. the hardness ratio may be somewhat lower than at higher intensities (Corbel et al. 2008; Cabanac et al. 2009). Since the sources spend most of their time in this state, the PRCB mechanism has enough time to establish a sufficiently strong magnetic field to permit the ejection of a jet. This may not be the case though for all black-hole XRBs in the quiescent state. For example, for GRO J1655-40 and XTE J1550-564, upper limits on the radio emission in the quiescent state were placed (Calvelo et al. 2010). In the source V404 Cyg, the radio emission is, in contrast, stronger than expected in the quiescent state (Gallo et al. 2005). As a rule 
however, black-hole XRBs exhibit a radio jet in the quiescent state and the PRCB can account for it.

In a typical outburst, as the X-ray luminosity increases, the hardness ratio remains approximately constant, and in the HID the sources trace the vertical line of the " $q$ "-shaped curve (region marked as hard state in Fig. 1). A steady, compact, partially selfabsorbed jet (first imaged in Cyg X-1 by Stirling et al. 2001) is present in this part of the "q"-shaped curve (region B in Fig. 1) and its radio luminosity $L_{\mathrm{R}}$ also increases in correlation with the X-ray luminosity $L_{\mathrm{X}}$ as $L_{\mathrm{R}} \propto L_{\mathrm{X}}^{0.71 \pm 0.1}$ (Corbel et al. 2000) or more accurately as $L_{\mathrm{R}} \propto L_{\mathrm{X}}^{0.6} M^{0.8}$, where $M$ is the mass of the black hole (Merloni et al. 2003; Falcke et al. 2004). The jet is mildly relativistic with a bulk Lorentz factor $\gamma<2$ (Fender et al. 2004a). Since the hard X-rays dominate the luminosity, this state is called hard. In addition, because the accretion disk is radiatively inefficient, it is generally believed to have an advection dominated accretion flow (ADAF) (Narayan \& Yi 1994, 1995) in its inner part (Gierlinski et al. 2008; Cabanac et al. 2009). The ADAF is geometrically thick, optically thin, and quite hot. The $\mathrm{ADAF}$ and the jet are the most likely origins of the hard X-ray spectrum (Giannios 2005; Markoff et al. 2005). As the X-ray luminosity of the sources increases, the electric current produced by the PRCB in the inner ADAF and the magnetic field in the steady jet increase. Thus, the radio emission also increases (e.g., Giannios 2005). It is then unsurprising that the radio luminosity and the X-ray luminosity are correlated.

As the sources reach the upper right part of the " $q$ "-shaped curve (region B in Fig. 1), the X-ray luminosity is at its maximum and the steady jet is at full strength. This is because the inner part of the accretion disk is occupied by the ADAF and the geometrically thin disk extends from some radius outwards (Cabanac et al. 2009). The PRCB works at its highest efficiency.

The hard state and the steady jet persist as the sources trace the upper right part of the " $q$ "-shaped curve (region to the left of $B$ in Fig. 1, marked as the hard intermediate state). The spectrum softens somewhat, but little else changes.

At more or less constant intensity, the hardness ratio then decreases further during the outburst and approaches the so-called jet line, which is the line between the existence and the nonexistence of a compact jet. As the source approaches the jet line, the radio emission peaks and the jet properties change drastically (Fender et al. 2009). The emission is no longer steady but eruptive, emitting discrete blobs, and its bulk Lorentz factor increases to $\gamma>2$. The high radio flares occur in most sources for extended periods of time (GX 339-4 is a notable exception) and are also associated with strong X-ray flaring (Brocksopp et al. 2002; Fender et al. 2009). The physical picture we have in mind is the following: as the outburst evolves, $\dot{m}$ apparently increases, the geometrically thin disk extends further in, less room is left for the ADAF, and the ADAF gradually disappears. As the sources reach the jet line, the geometrically thin disk extends all the way to the ISCO. Regardless of the magnetic field that existed inside the ISCO of the geometrically thick ADAF will now have trouble being held there by the thin disk. We propose that the thin disk becomes unstable to non-axisymmetric magnetic "Rayleigh-Taylor-type" instability modes, and the accumulated magnetic field escapes to the outer disk in the form of magnetized "strands" 3 . Such an instability may explain naturally the

\footnotetext{
3 While in the ADAF stage, magnetic pressure is balanced by the infall ram pressure (Eq. (4)). After plasma cooling and the collapse of the disk to $h / r \lesssim 10^{-1}$, the magnetic stresses at the inner edge $B_{z} \mathrm{~d} B_{\mathrm{r}} / \mathrm{d} z \sim$ $B_{z} B_{r \text {,surface }} / h \sim B_{z}^{2} / h$ become too strong for the thin disk to contain the field and instabilities are expected to set in.
}

flaring nature of the jets as the sources approach the jet line during the hard-to-soft transition.

To the left of the jet line (regions C and D in Fig. 1), the sources are in the so-called soft state, the accretion disk is widely believed to be of the Shakura-Sunyaev (1973) type, i.e., geometrically thin and optically thick, and extends all the way to the ISCO. In this state, radio emission is in nearly all cases either undetectable or optically thin, consistent with the disappearance of the compact, partially optically thick, steady jet that was present in the hard state (Fender et al. 2009). The black-hole XRB H1743-322 provides the tightest constraints to date for radio quenching in the soft state (a jet quenching factor of $\sim 700$; Coriat et al. 2011). The PRCB works in this state also, but since the thickness of the disk $h$ is much smaller than the radius $r$ of the ISCO, the efficiency of the battery is significantly lower. For example, for $h / r \approx 10^{-1}$, the timescale for the establishment of an equipartition magnetic field at the inner part of the thin disk (Eq. (8) above) is on the order of five months at the upper left part of the "q"-shaped curve (region C in Fig. 1) and becomes even longer as the intensity of the source decreases (transition from region $\mathrm{C}$ to region $\mathrm{D}$ in Fig. 1) ${ }^{4}$. This timescale is substantially longer than the characteristic time that the sources spend in the various parts in-between regions $C$ and $D$ in Fig. 1.

Some sources, including the archetypal black-hole XRB GX 339-4, cross the jet line only once (Fender et al. 2009). While in the soft state, they may trace small closed loops (see, e.g., Fig. 7 of Fender et al. 2004a). No jet has ever been detected when the sources trace these loops. However, when a source does decide to go back and forth and thus to cross the jet line leisurely several times, then a) a steady jet is created as the hardness ratio increases (soft-to-hard state transition - the inner accretion disk gradually thickens, the magnetic field is built at an accelerated rate, and the disk becomes stable to non-axisymmetric RaleighTaylor modes); b) a flaring jet appears as the sources return to the jet line (hard-to-soft state transition - the inner disk becomes thinner, and the accumulated field makes the thinner disk unstable); and c) just as in the first crossing of the jet line, the jet disappears in the soft state. This is exactly what is observed in GRS 1915+105 (Rushton et al. 2010; see also Brocksopp et al. 2002 for XTE J1859+226). This source is known to be in the soft state for long periods of time, although it makes repeated excursions to the hard state.

Exactly the opposite seems to occur in Cyg X-1 (Rushton et al. 2011). This source is known to be in the hard state for long periods of time (Di Salvo et al. 2001), although it makes repeated attempts ("failed" state transitions; Pottschmidt et al. 2003) to reach the soft state, where it did exist at least once (Fender et al. 2006).

To complete the " $q$ "-shaped curve, the sources in the decay phase of the outburst increase their hardness ratio at about constant intensity and cross the jet line from the left (region marked hard intermediate state, to the left of E in Fig. 1). The intensity at this crossing (soft-to-hard state transition) is significantly lower than in the previous crossing (hard-to-soft transition). The accretion flow again becomes ADAF, initially at the outer part and then at a larger extent, and the PRCB now operates very efficiently. As a result, a jet gradually forms (Brocksopp et al. 2005; Fender et al. 2009), and by the time that the sources reach region E of Fig. 1, a steady jet is established and persists as the intensity declines and the sources transit to the quiescent state (region A of Fig. 1).

\footnotetext{
4 From Eqs. (5) and (7), the timescale for field growth is inversely proportional to the XRB X-ray luminosity.
} 


\subsection{Jets in neutron-star $X$-ray binaries}

Low-magnetic-field neutron-star XRBs are divided into two distinct classes, based on their X-ray spectral and timing properties. They are named atoll and $\mathrm{Z}$ sources (Hasinger \& van der Klis 1989) because of the shape that they trace in a color-color diagram. Both classes exhibit jets, but because their radio emission is weaker than that in black-hole XRBs, they have received less attention (for comprehensive reviews of the properties of jets from neutron-star XRBs, see Migliari \& Fender 2006 and Körding et al. 2006). Moreover, neutron stars are more complex than black holes in that they possess a solid surface and a stellar magnetic field. For the above reasons, our discussion of jets in neutron-star XRBs here is more tentative and qualitative than the discussion of Sect. 3.1.

In the HID, neutron-star XRBs trace a "q"-shaped curve, similar to that of black-hole XRBs (Maitra \& Bailyn 2004). Nevertheless, there are two major differences between the jets in these two types of binaries:

1. The radio luminosities of neutron-star jets are typically 30 times lower than those of black-hole jets at comparable X-ray luminosities (Fender \& Kuulkers 2001; Migliari et al. 2003).

2. Radio emission has been detected in the soft state of some neutron-star XRBs (Migliari et al. 2004), as opposed to their non-detection in black-hole sources, when they are in the same state.

Both of these differences can be explained physically using the PRCB along with the fact that neutron stars have a solid surface. Unlike in black-hole XRBs, where the accretion disk extends inward to the ISCO, in neutron-star XRBs with weak magnetic fields the accretion disks extend all the way to the surfaces of the stars because in this case, the ISCO practically coincides with the stellar surface.

One of the well-studied neutron-star binaries in both radio and X-rays is the atoll source Aql X-1. The evolution of the radio and the $\mathrm{X}$-ray emission of this source throughout an entire outburst was reported by Miller-Jones et al. (2010). In the HID, Aql X-1 traces a "q"-shaped curve, similar to that of black-hole XRBs. In the hard state, the source exhibits a compact jet, similar to those of black-hole XRBs, but with weaker radio emission. The inner part of the accretion disk is of the ADAF form, as in black-hole XRBs, and thus the PRCB operates in some capacity. The difference is that now the solid surface of the neutron star blocks the radiation from the opposite side of the inner accretion disk. Thus, the PRCB now operates at a reduced efficiency. In addition, the radio emission is weaker for the following important reason. The X-ray luminosity scales as $L_{\mathrm{X}}(N S) \propto \dot{m} M$ for all neutron-star states and as $L_{\mathrm{X}}(B H) \propto \dot{m}^{2} M$ for black holes accreting in the ADAF state (Narayan et al. 1997; Abramowicz \& Fragile 2011), whereas the radio luminosity scales as $L_{\mathrm{R}} \propto \dot{m} M$ in both cases; hence for accreting sources of comparable Xray luminosities, the ratio of their jet (radio) powers will be $L_{\mathrm{R}}(B H) / L_{\mathrm{R}}(N S) \simeq \dot{m}^{-1}$.

Although neutron-star XRBs can produce ultra-relativistic outflows in some cases (Fender et al. 2004b), their transition from the hard to the soft state generally occurs in a much smoother way than in black-hole XRBs (Miller-Jones et al. 2010). This can also be understood physically by the PRCB continuing to operate, albeit at a lower efficiency, when the neutronstar sources transit from the hard to the soft state.

In the soft state, the jet of Aql X-1 is quenched. This is expected because in the soft state the inner accretion disk is thinner
(Shakura-Sunyaev type), and this part of the disk as well as the region where accreting matter impinges onto the neutron-star surface (Inogamov \& Sunyaev 2010) are both optically thick. This implies that there is little difference in the azimuthal velocities between the points of emission and scattering of the radiation. Thus, the PRCB is quite inefficient.

Unlike black-hole XRBs, radio emission has been detected in some cases in the soft state of neutron-star XRBs (Migliari et al. 2004). These cases can be understood as follows. In the area where the accreted material impinges onto the neutron-star surface, a "spreading layer" likely forms with scale height much larger than the actual thickness of the accretion disk (Inogamov \& Sunyaev 2010). Radiation from this "spreading layer" then hits the accretion disk from above and below, thus fueling the PRCB. The battery operates in this case too, though at a lower efficiency than for when the inner part of the accretion disk is of the ADAF type. (Here we have made the tacit assumption that there is a non-negligible difference between the spin frequency of the neutron star and the Keplerian frequency of the disk.)

\subsection{Additional remarks}

X-ray pulsars do not exhibit compact jets despite their strong magnetic fields (a few times $10^{12} \mathrm{G}$ ). In the context of the PRCB, this can be understood as follows. The accretion disks extend inwards to the Alfvén radius, which is on the order of $10^{8} \mathrm{~cm}$ (Ghosh \& Lamb 1978). At this radius, and because the accretion disks are geometrically thin, the photon flux at the inner edges of the disks is very low and the PRCB is thus very inefficient. In addition, the accretion flow distorts the overall dipolar geometry of the magnetic flux generated by the PRCB since, in an X-ray pulsar, accretion does not proceed radially toward the center, but follows magnetic field lines toward the magnetic poles.

However, it is unclear what the magnetic-field strength is in atoll and $\mathrm{Z}$ sources. It is generally thought to be low because the discovery (Wijnands \& van der Klis 1998) of coherent $401 \mathrm{~Hz}$ X-ray pulsations from the accretion-powered pulsar SAX J1808.4-3658 allowed the determination (Psaltis \& Chakrabarty 1999) of a surface magnetic field of $10^{8}-10^{9} \mathrm{G}$ in this source. However, it is unknown whether other atoll and $\mathrm{Z}$ sources have surface magnetic fields of this order of magnitude; their magnetic fields could very well be weaker.

If the surface magnetic field of neutron stars is weaker than the equipartition value given by Eq. (4) above, then the PRCB operates effectively. On the other hand, if the surface magnetic field is stronger than the equipartition value, this strong magnetic field determines where the inner disk will be truncated and whether a jet will be produced. Judging from the observed X-ray pulsars, it seems that a significant surface magnetic field inhibits jet formation.

Along the same line of reasoning, it is unsurprising that SS Cyg, a non-magnetic white-dwarf XRB, exhibits a "q"-shaped curve in the HID and jet emission characteristics similar to the atoll source Aql X-1 and the black-hole XRB GX 339-4 (Körding et al. 2008). Thus, it appears very likely that atoll and $\mathrm{Z}$ sources, as a class, have very weak magnetic fields.

\section{Conclusions}

Not only is the PRCB able to explain the poloidal magnetic fields required for the ejection of jets in white-dwarf, neutronstar, and black-hole XRBs, but it also provides a physical framework through which we can understand many of the jet manifestations in these sources in relation to their spectral states. 
Equally importantly, the PRCB provides naturally timescales for these transitions that are much longer than the dynamical ones and in general agreement with those observed. Our understanding is semi-quantitative at this point, but detailed calculations are underway and will be reported elsewhere.

In view of our proposed picture, we make the following predictions about black-hole XRBs:

1. The transition from the hard to the soft X-ray state (first jet-line crossing in the HID) will always be associated with eruptive jet emission, flaring, and the disappearance of the compact jet past the jet line.

2. The transition from the soft to the hard X-ray state (last jetline crossing in the HID) will always be associated with the formation of a steady jet within 1-5 days of the crossing. No eruptive emission will ever be seen in such a one-way crossing.

In the case of neutron-star XRBs, our predictions are the following:

1. No compact jet will be formed in normal X-ray pulsars (magnetic field $\gtrsim 10^{12} \mathrm{G}$ ).

2. No jet will be formed in the soft state of atoll and Z sources if the spin frequency of the neutron star is comparable to the Keplerian frequency of the inner part of the accretion disk.

3. In atoll and $\mathrm{Z}$ sources, the larger the difference between the neutron-star spin frequency and the Keplerian frequency of the inner part of the accretion disk, the stronger the radio jet in the soft state .

Acknowledgements. This research has been supported in part by EU Marie Curie project No. 39965, and EU REGPOT project number 206469. N.D.K. thanks Tomaso Belloni for providing hardness-intensity data of GX 339-4 during various outbursts and Michiel van der Klis and Dimitrios Psaltis for useful discussions concerning millisecond pulsars. I.C. thanks Maxim Lyutikov, Ramesh Narayan, Roman Shcherbakov, and Sasha Tchekhovskoy for discussions concerning accretion disks and the PRCB.

\section{References}

Abramowicz, M. A., \& Fragile, P. C. 2011, Liv. Rev. Relat., submitted [arXiv: 1104.5499]

Belloni, T. 2010, LNP, 794, 53

Blandford, R. D., \& Begelman, M. C. 2004, MNRAS, 349, 68

Blandford, R. D., \& Payne, D. G. 1982, MNRAS, 199, 883

Brocksopp, C., Fender, P. R., McCollough, M., et al. 2002, MNRAS, 331, 765

Brocksopp, C., Corbell, S., Fender, R. P., et al. 2005, MNRAS, 356, 125

Cabanac, C., Fender, R. P., Dunn, R. J. H., \& Körding, E. G. 2009, MNRAS, 404, 738

Calvelo, D. E., Fender, R. P., Russell, D. M., et al. 2010, MNRAS, 409, 839

Christodoulou, D. M., Contopoulos, I., \& Kazanas, D. 2008, ApJ, 674, 388

Christodoulou, D. M., Gabuzda, D. C., Contopoulos, I., \& Kazanas, D. 2011, A\&A, submitted

Contopoulos, J. 1995, ApJ, 450, 616

Contopoulos, I., \& Kazanas, D. 1998, ApJ, 508, 859

Contopoulos, I., Kazanas, D., \& Christodoulou, D. M. 2006, ApJ, 652, 1451

Contopoulos, I., Christodoulou, D. M., Kazanas, D., \& Gabuzda, D. C. 2009, ApJ, 702, L148

Corbel, S., Fender, R. P., Tzioumis, A. K., et al. 2000, A\&A, 359, 251

Corbel, S., Körding, E., \& Kaaret, P. 2008, MNRAS, 389, 1697

Coriat, M., Corbel, S., Prat, L., et al. 2011, MNRAS, 414, 677
Di Salvo, T., Done, C., Zycki, P. T., Burderi, L., \& Robba, N. R. 2001, ApJ, 547, 1024

Done, C., Gierliński, M., \& Kubota, A. 2007, A\&AR, 15, 1

Falcke, H., Körding, E., \& Markoff, S. 2004, A\&A, 414, 895

Fender, R. P., \& Kuulkers, E. 2001, MNRAS, 324, 923

Fender, R. P., Belloni, T. M., \& Gallo, E. 2004a, MNRAS, 355, 1105

Fender, R., Wu, K., Johnston, H., et al. 2004b, Nature, 427, 222

Fender, R. P., Stirling, A. M., Spencer, R. E., et al. 2006, MNRAS, 369, 603

Fender, R. P., Homan, J., \& Belloni, T. M. 2009, MNRAS, 396, 1370

Fereira, J., Petrucci, P.-O., Henri, G., Saugé, L., \& Pelletier, G. 2006, A\&A, 447, 813

Fragile, P. C., \& Meier, D. L. 2009, ApJ, 693, 771

Gallo, E., Fender, R. P., \& Hynes, R. I. 2005, MNRAS, 356, 1017

Gallo, E., Fender, R. P., Miller-Jones, J. C. A., et al. 2006, MNRAS, 370, 1351

Giannios, D. 2005, A\&A, 437, 1007

Gierlinski, M., Done, C., \& Page, K. 2008, MNRAS, 388, 753

Ghosh, P., \& Lamb, F. K. 1978, ApJ, 223, L83

Hameury, J.-M., Barret, D., Lasota, J.-P., et al. 2003, A\&A, 399, 631

Hasinger, G., \& van der Klis, M. 1989, A\&A, 225, 79

Hawley, J. F. 2009, Ap\&SS, 320, 107

Homan, J., \& Belloni, T. 2005, Ap\&SS, 300, 107

Igumenshchev, I. V. 2008, ApJ, 677, 317

Inogamov, N. A., \& Sunyaev, R. A. 2010, Astron. Lett., 36, 848

Kong, A. K. H., McClintock, J. E., Garcia, M. R., Murray, S. S., \& Barret, D. 2002, ApJ, 570, 277

Körding, E. G., Fender, R. P., \& Migliari, S. 2006, MNRAS, 369, 1451

Körding, E., Rupen, M., Knigge, C., et al. 2008, Science, 320, 1318

Lovelace, R. V. E., Rothstein, D. M., \& Bisnovatyi-Kogan, G. S. 2009, ApJ, 701, 885

Lubow, S. H., Papaloizou, J. C. B., \& Pringle, J. E. 1994, MNRAS, 267, 235

Lynden-Bell, D. 1996, MNRAS, 279, 389L

Machida, M., Nakamura, K., \& Matsumoto, R. 2006, PASJ, 58, 193

Maitra, D., \& Bailyn, C. D. 2004, ApJ, 608, 444

Markoff, S. 2010, LNP, 794, 143

Markoff, S., Nowak, M. A., \& Wilms, J. 2005, ApJ, 635, 1203

Meier, D. L. 2001, ApJ, 548, L9

Meier, D. L. 2005, Ap\&SS, 300, 55

Merloni, A., Heinz, S., \& di Matteo, T. 2003, MNRAS, 345, 1057

Migliari, S., \& Fender, R. P. 2006, MNRAS, 366, 79

Migliari, S., Fender, R. P., Rupen, M., et al. 2003, MNRAS, 342, L67

Migliari, S., Fender, R. P., Rupen, M., et al. 2004, MNRAS, 351, 186

Migliari, S., Miller-Jones, J. C. A., Fender, R. P., et al. 2007, ApJ, 671, 706

Migliari, S., Tomsick, J. A., Miller-Jones, J. C. A., et al. 2010, ApJ, 710, 117

Migliari, S., Miller-Jones, J. C. A., \& Russell, D. M. 2011, MNRAS, 415, 2407

Mignonge, A., Rossi, P., Bodo, G., Ferrari, A., \& Massaglia, S. 2010, MNRAS, 402, 7

Miller-Jones, J. C. A., Sivakoff, G. R., Altamirano, D., et al. 2010, ApJ, 716, L109

Miyamoto, S., Kitamoto, S., Hayashida, K., \& Egoshi, W. 1995, ApJ, 442, L13

Narayan, R., \& Yi, I. 1994, ApJ, 428, L13

Narayan, R., \& Yi, I. 1995, ApJ, 452, 710

Narayan, R., Garcia, M. R., \& McClintock, J. E. 1997, ApJ, 478, L79

Pottschmidt, K., Wilms, J., Nowak, M. A., et al. 2003, A\&A, 407, 1039

Psaltis, D., \& Chakrabarty, D. 1999, ApJ, 521, 332

Remillard, R. A., \& McClintock, J. E. 2006, ARA\&A, 44, 49

Romanova, M. M., Ustyugova, G. V., Koldova, A. V., \& Lovelace, R. V. E. 2009, in Protostellar Jets in Contect, ed. K. Tsinganos, O. Ray, \& M. Stute, Ap\&SS Proc. (Berlin: Springer), 153

Rushton, A., Spencer, R., Fender, R., \& Pooley, G. 2010, A\&A, 524, A29

Rushton, A., Miller-Jones, J., Paragi, Z., et al. 2011 [arXiv: 1101.3322v1]

Shakura, N. I., \& Sunyaev, R. A. 1973, A\&A, 24, 337

Spruit, H. C. 2010, LNP, 794, 233

Stiele, H., Muñoz-Darias, T., Motta, S., \& Belloni, T. 2011, 25th Texas Symposium on Relativistic Astrophysics, Texas 2010 [arXiv: 1103.4312]

Stirling, A. M., Spencer, R. E., de la Force, C. J., et al. 2001, MNRAS, 327, 1273

Tchekhovskoy, A., Narayan, R., \& McKinney, J. C. 2011, MNRAS, 418, L79

Tudose, V., Fender, R. P., Linares, M., Maitra, D., \& van der Klis, M. 2009, MNRAS, 400, 2111

Wijnands, R., \& van der Klis, M. 1998, Nature, 394, 344

5 This prediction was recently confirmed by Migliari et al. (2011). 